\title{
3D ultrasound-guided resection of low-grade gliomas: principles and clinical examples
}

\author{
Geirmund Unsgård, MD, PhD, 1,2 and Frank Lindseth, $\mathrm{PhD}^{2,3}$ \\ ${ }^{1}$ Department of Neurosurgery, St. Olav University Hospital; ${ }^{2}$ Department of Neuromedicine and Movement Science, \\ Norwegian University of Science and Technology; and ${ }^{3}$ Department of Computer Science, SINTEF, Trondheim, Norway
}

3D ultrasound (US) is a convenient tool for guiding the resection of low-grade gliomas, seemingly without deterioration in patients' quality of life. This article offers an update of the intraoperative workflow and the general principles behind the 3D US acquisition of high-quality images.

The authors also provide case examples illustrating the technique in two small mesial temporal lobe lesions and in one insular glioma. Due to the ease of acquiring new images for navigation, the operations can be guided by updated image volumes throughout the entire course of surgery. The high accuracy offered by 3D US systems, based on nearly realtime images, allows for precise and safe resections. This is especially useful when an operation is performed through very narrow transcortical corridors.

https://thejns.org/doi/abs/10.3171/2019.9.FOCUS19605

KEYWORDS navigated ultrasound; 3D ultrasound; LGG; surgical setup

$\mathrm{L}$ OW-GRADE gliomas (LGGs) are slow-growing tumors prone to malignant transformation. ${ }^{7,13,18} \mathrm{Re}$ section is the primary treatment for LGGs since it delays malignant transformation and prolongs a patient's survival.,18 Often, LGGs represent a surgical challenge due to involvement of eloquent parts of the brain, ${ }^{4,7}$ which is particularly worrisome in LGGs because it is often difficult to differentiate the tumor from normal tissue during resection.

We have demonstrated that when the resection is guided by $3 \mathrm{D}$ ultrasound (US), the improved tumor control gained during the resection does not come at the cost of deterioration in a patient's quality of life. ${ }^{9}$

In this article, we provide both general information and specific examples of how to use 3D US in the treatment of challenging LGGs.

\section{Methods}

\section{General Principles}

We routinely perform preoperative 3D MRI 1-3 days before surgery, and in eloquent cases we add functional MRI and/or tractography as needed. ${ }^{2}$ We have used a neuronavigation system with an integrated 3D US scanner, which enables simultaneous navigation in both MRI and US volumes (SonoWand Invite). In this intraoperative imaging system, the volumes are displayed as 2D slices steered by a pointer or different surgical equipment, either as oblique anyplane slices, as axial-coronal-sagittal slices, or as tip views. The tip view displays an image plane perpendicular to a pointer or navigated instrument through its tip or through the focus point of the navigated microscope.

Many US probes are not sterilizable and have to be draped. Air caps in front of the probes must be avoided as air prevents propagation of the US waves. The initial image acquisition that is done before opening of dura mater may be hampered by calcification in dura, giving rise to artifacts. Thus, it is advisable to acquire new images after dural opening. In US images, LGGs appear hyperechoic compared to the normal brain due to their higher cellularity. Linear probes in the range $8-12 \mathrm{MHz}$ and with a dynamic focus produce excellent image quality from the first millimeter down to a depth of approximately $5 \mathrm{~cm}$, while a 4- to 8-MHz probe is best for very deep-seated lesions. The rectangular image of a linear probe can be too narrow in the deeper part of a large tumor, but this can be compensated for using a "wide application" that depicts the brain in a cone-shaped way. To be able to obtain good image quality throughout the operation, it is mandatory 
that the patient is positioned in a way that enables vertical access to the lesion. In that way, the coupling fluid (saline or Ringer's solution) will stay in the cavity during image acquisition. The vertical surgical corridor also limits the need for the application of traction with spatulas that may harm the brain and worsen brain shift. Our experience is that the vertical access can nearly always be obtained. The only situation in which it is not possible to use US is when operating in the posterior fossa with the patient in a sitting position. Furthermore, it is important to acquire US images that also include normal tissue to facilitate the interpretation of tumor borders.

\section{Enhancement Artifacts}

The image quality may deteriorate toward the end of an operation because of the bright rim or enhancement artifacts. These artifacts are caused by the higher attenuation of sound waves in tissue than in the saline/Ringer's solution that fills the operative cavity to obtain acoustical coupling, and they are seen especially in operations that involve larger cavities. ${ }^{16}$ Image interpretation toward the end of the procedure is generally easier if there are several image updates during the previous part of the surgery. A small probe placed into the cavity may also reduce these artifacts, but it may be troublesome to use.

\section{Guided Resection With the Tracked Ultrasound Aspirator}

It may be difficult to decide what is LGG tissue and what is normal tissue based on texture and the microscopic image. We, therefore, rely on the US images to a large extent. To perform image-guided resection, we always attach a tracking frame to the US aspirator (Videos 1 and 2), and then we calibrate the tracked Cavitron US aspirator (CUSA) to the intraoperative imaging system.

VIDEO 1. Image-guided resection, patient 1. Copyright Norwegian National Advisory Unit for Ultrasound and Image-Guided Therapy. Published with permission. Click here to view.

VIDEO 2. Image-guided resection, patient 3. Copyright Norwegian National Advisory Unit for Ultrasound and Image-Guided Therapy.

Published with permission. Click here to view.

The resection is done by looking in the microscope and glancing at the navigation monitor. At the beginning of the resection, it is useful to display 3D MR images along with 3D US images to help in the interpretation of the US images. Removal of the tumor tissue is done from inside the tumor out to the tumor border. When we approach the tumor border, a new 3D US volume is acquired to do the last part of the resection as precisely as possible. Before acquiring new images, hemostasis is established. If spatulas are used, they are removed together with hemostatic material and patties, and the cavity is filled with saline/ Ringer's solution. This process typically takes a few minutes, and owing to the minimal disruption of the surgical workflow, we usually acquire several 3D US volumes during the operation, and the resection is always based on the latest volume. At the end of the resection, we acquire a final volume for resection control.

\section{Stimulation}

Mapping by subcortical stimulation is done with a
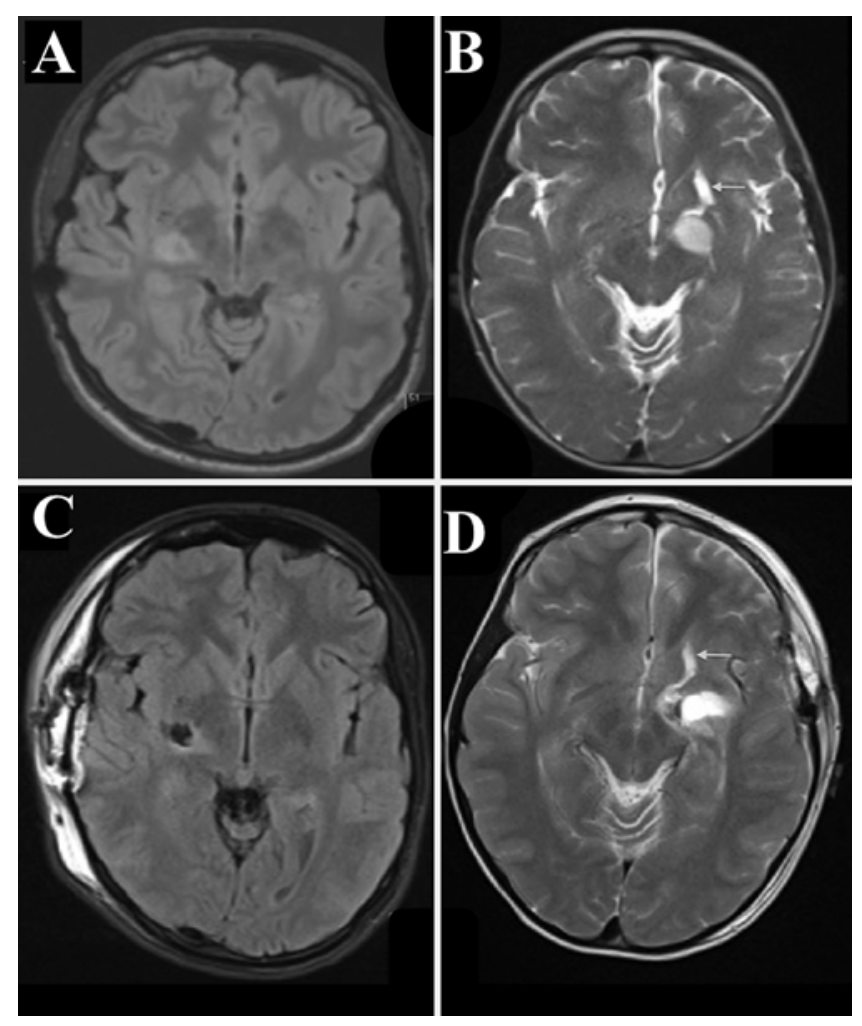

FIG. 1. A: Preoperative FLAIR MR image of patient 1. B: Preoperative T2-weighted MR image of patient 2. The injury in front of the tumor (arrow) is a scar from the previous surgery performed via the transsylvian approach. C: Image of patient 1 obtained 1 day after the operation. D: Image of patient 2 obtained 1 day after the operation. The arrow points to the same scar as mentioned in $\mathrm{B}$.

monopolar probe using the ISIS IOM System (inomed). Motor mapping can provide information about the distance from the motor tracts. ${ }^{10} \mathrm{~A}$ threshold of $3 \mathrm{~mA}$ has been found to be the cut-off point below which irreversible damage to the motor tract may occur. ${ }^{12,15}$

\section{Results}

\section{Clinical Examples}

Informed consent was obtained from the patients described in the following 3 cases. All procedures were performed in accordance with the ethical standards of the 1964 Helsinki Declaration and its later amendments or comparable ethical standards.

Patients 1 and 2 had very similar lesions (Fig. 1A and B). The lesions were in the corpus amygdala, extending superiorly in close proximity to the globus pallidus and consequently involving the temporal stem with its uncinate and inferior frontooccipital fascicle, in addition to being in close proximity to the optic tract and internal capsule. .11,14,22 $^{2}$

Patient 1, a 19-year-old man, had longstanding troubles with fear and fatigue, and he had dropped out of high school. MRI 6 months prior to referral to our department demonstrated a small lesion suspected to be an LGG involving the right amygdala with a superior extension (Fig. 1A). The optic tract could not be distinguished from the 

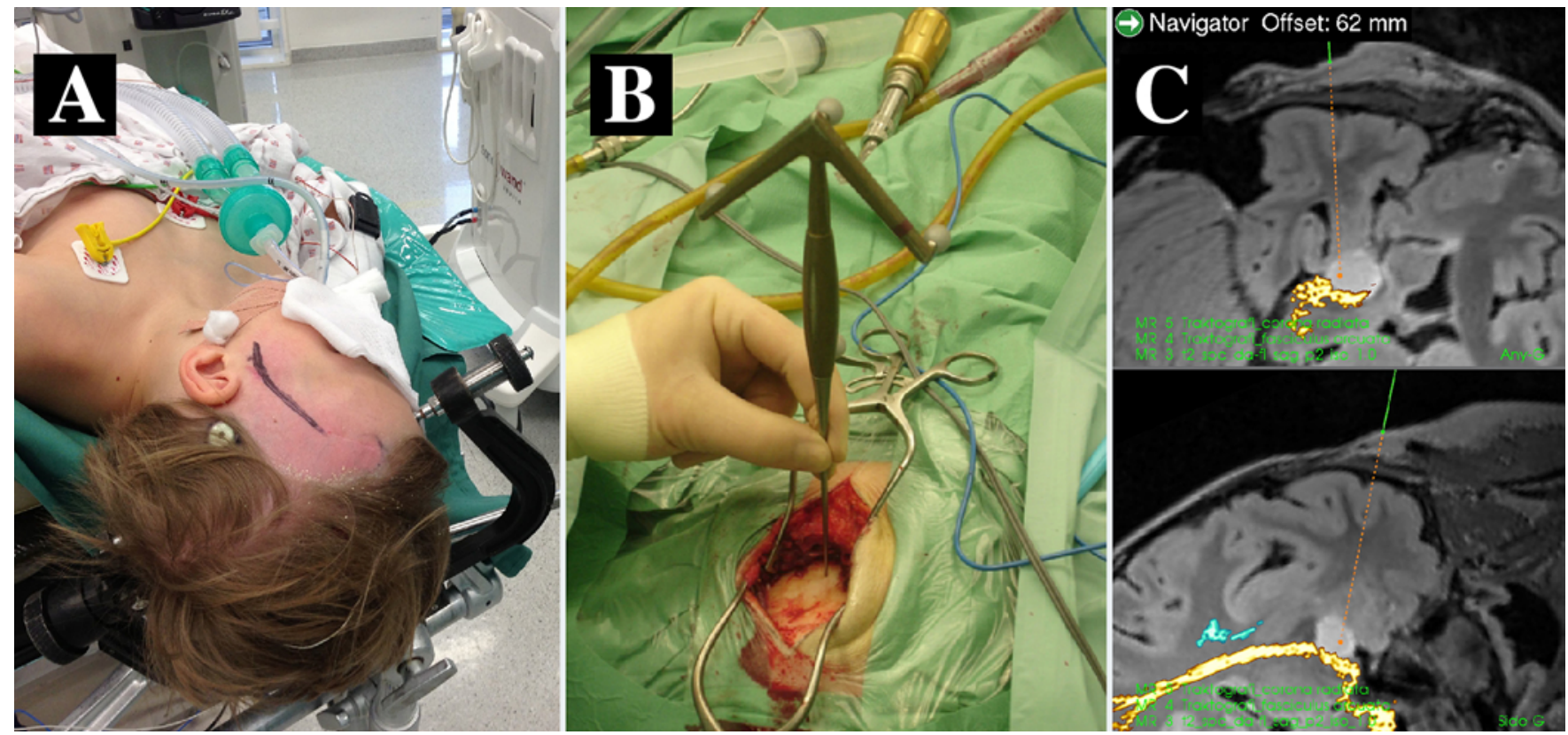

FIG. 2. A: Positioning of the patient. B: Opening of the skin and splitting of the muscle. C: Navigation planning of a transcortical trajectory using preoperative MR images registered to the patient, and with diffusion tensor imaging of the pyramidal tract (yellow) fused into the MRI volume. The two images are reformatted and displayed as perpendicular planes oriented according to the position of the navigated pointer. The position of the pointer in B is displayed as green lines with virtual extensions (orange dotted lines) in the two images in $\mathrm{C}$.

lower limit of the tumor. The pyramidal tract was in close proximity medially. A navigation-assisted biopsy did not give a clear answer. The patient and his family strongly wanted a resection of the lesion despite the surgical risks.

Patient 2, a 14-year-old boy, had had, for many years, mental difficulties and had been given the diagnosis of attention-deficit/hyperactivity disorder. Three years prior to referral to our department, MRI revealed a lesion in the left amygdala suspected to be LGG. The patient was followed with regular MRI, and because of growth in the lesion, he underwent surgery at his local university hospital; the procedure was performed via a transsylvian approach and assisted by conventional neuronavigation. No tumor was found. On postoperative MRI, it seemed as though the biopsy specimens had been acquired outside the lesion (Fig. 1B). Because of our experience with 3D US-guided surgery, the patient was referred to our hospital.

In both cases, we performed preoperative 3D MRI with tractography of the pyramidal and optical tracts 2 days before the operation. Unfortunately, the optic tract could not be visualized in these two patients.

Patient 3, a 50-year-old woman, had for some time felt tired and had become so sensitive to sounds that she was on sick leave. MRI showed a suspected LGG in the left (dominant) insula region. She wanted to undergo surgery to have her tumor removed.

\section{Surgical Setup and Intraoperative Imaging}

The surgical setups for patients 1 and 2 represent the standard approach we use to access mesial temporal lobe pathological entities. The patients were placed in a supine position with their heads parallel to the floor (Fig. 2A) and tilted about $15^{\circ}$ to be able to access the lesion via a vertical transcortical approach, preferably through the inferior temporal gyrus. Appropriate positioning was verified by MRI navigation (Fig. 2C).

The skin incision was a temporal straight line, about 4-5 cm, down to the zygomatic arch (Fig. 2A). The fascia and the temporalis muscle were split and held apart by a self-holding retractor, which enables an ellipsoid craniotomy down to the zygomatic arch, about $4 \times 5 \mathrm{~cm}$ (Fig. $2 B)$. Immediately after making the craniotomy, a 3D US volume image was acquired using an 11-MHz linear array probe (width $3.2 \mathrm{~cm}$ ) and the "wide application" (Fig. 3). Thereafter, the surgery was guided by the 3D US volume (Video 1). Navigated free-hand biopsies for a frozen section were sampled. To find an appropriate trajectory from the lower temporal gyrus through the temporal lobe, we use the navigated microscope interface. The crosshairs in the navigated microscope were used to place small spatulas in an appropriate position to achieve a narrow surgical corridor. In both cases, the lesion was reached, and biopsies confirmed a low-grade astrocytoma. The tumor was resected using the navigated CUSA guided by the 3D US images (Fig. 3 and Video 1). Stimulation was used to ensure avoidance of the pyramidal tract.

For the insula approach, positioning of the patient was similar to that used in the mesial temporal approach except for the tilting. A standard temporal craniotomy and a transcortical access were used. The 3D US was used to select a direct approach to the middle of the tumor at the lower part of the superior gyrus of the temporal lobe. We tried to make the operating canal as narrow as possible by using small spatulas and a navigated microscope (Fig. 4). 

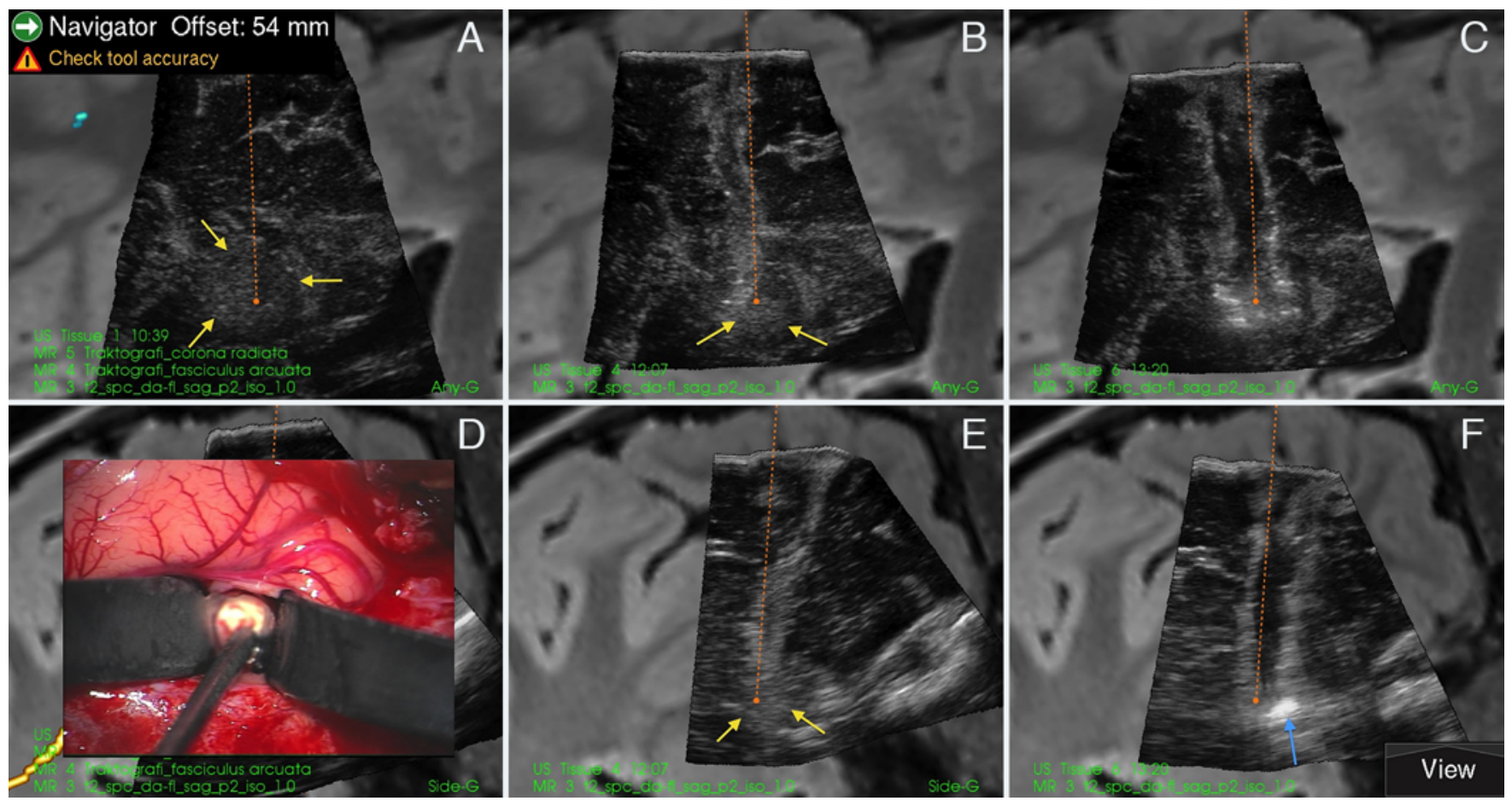

FIG. 3. Snapshots from the navigation monitor of patient 2 showing two perpendicular sections (dual anyplane, top and bottom rows) through the 3D US volumes acquired at 3 different stages of the operation and overlaying the corresponding MR image slices. A and D: The left column shows images from a US volume acquired before the start of resection. B and E: In the center column, a considerable part of the tumor has been removed. C and F: The right column shows US images obtained at the end of the resection. The yellow arrows point to the tumor borders. The end of the orange dotted line in the center column points to tumor tissue that has been removed in the left column (at the end of the operation). The hyperechoic signal in the lower image of the right column (blue arrow) is most likely a blood clot. The overlaid microscopic image shows the opening in the cortex.

The insula arteries were identified by Doppler angiography, and the tumor was resected between the insula arteries, guided by US and the tracked CUSA (Video 2). All patients were operated on while asleep under anesthesia.

\section{Surgical Outcomes}

For both patients 1 and 2, no neurological deficits could be detected on routine postoperative neurological examination, and no medical or surgical complications were noted. In both cases, a radiological gross-total resection was achieved (Fig. 1C and D).

An ophthalmological investigation of patient 1 three weeks after the operation showed completely normal visual fields. No immediate effect was seen on the patient's anxiety.

In patient 2 , ophthalmological assessment 8 weeks after the operation demonstrated a right-sided upper quadrantanopia with no subjective discomfort. A subtle semantic paraphasia was noted.

Patient 3, operated on for an insular glioma, woke up in splendid condition immediately after the operation and was able to go home 3 days later. Postoperative MRI showed gross-total resection.

\section{Discussion}

In this paper, we have provided practical information on how to carry out 3D US-guided surgery in patients with LGGs in order to achieve good image quality and consequently good surgical results. Our strategy was illustrated by describing 2 cases of LGG in the amygdala region and 1 case of an insular glioma.

During the last 2 decades "asleep" operations with 3D US guidance have been the preferred strategy for LGG procedures at our hospital. Intraoperative MRI has not been available for direct comparison. However, the clinical usefulness of 3D US in terms of clinical outcome following LGG surgery has been demonstrated in several publications..$^{7-9}$ In a recent publication, the effectiveness of 3D US, in terms of extent of resection, was concluded to be consistent with published studies using other advanced neurosurgical tools. ${ }^{3}$

A benefit of 3D US is the ease with which it is possible to obtain new images during a resection, which allows the surgeon to operate on updated and precise volumes at any time. It takes only a few minutes from the surgeon's decision to acquire a new image volume until the surgeon is ready to operate based on the new volume. The high accuracy and good image quality of LGGs provided by navigated 3D US are very useful because sometimes it is difficult to differentiate the LGG from the normal tissue when using the operative microscope. The navigated CUSA enables precise LGG resection even in deep-seated areas. The tip of the CUSA will steer the display of the cross sections on the monitor, and by glancing at the monitor, the surgeon will know the position of the CUSA tip 

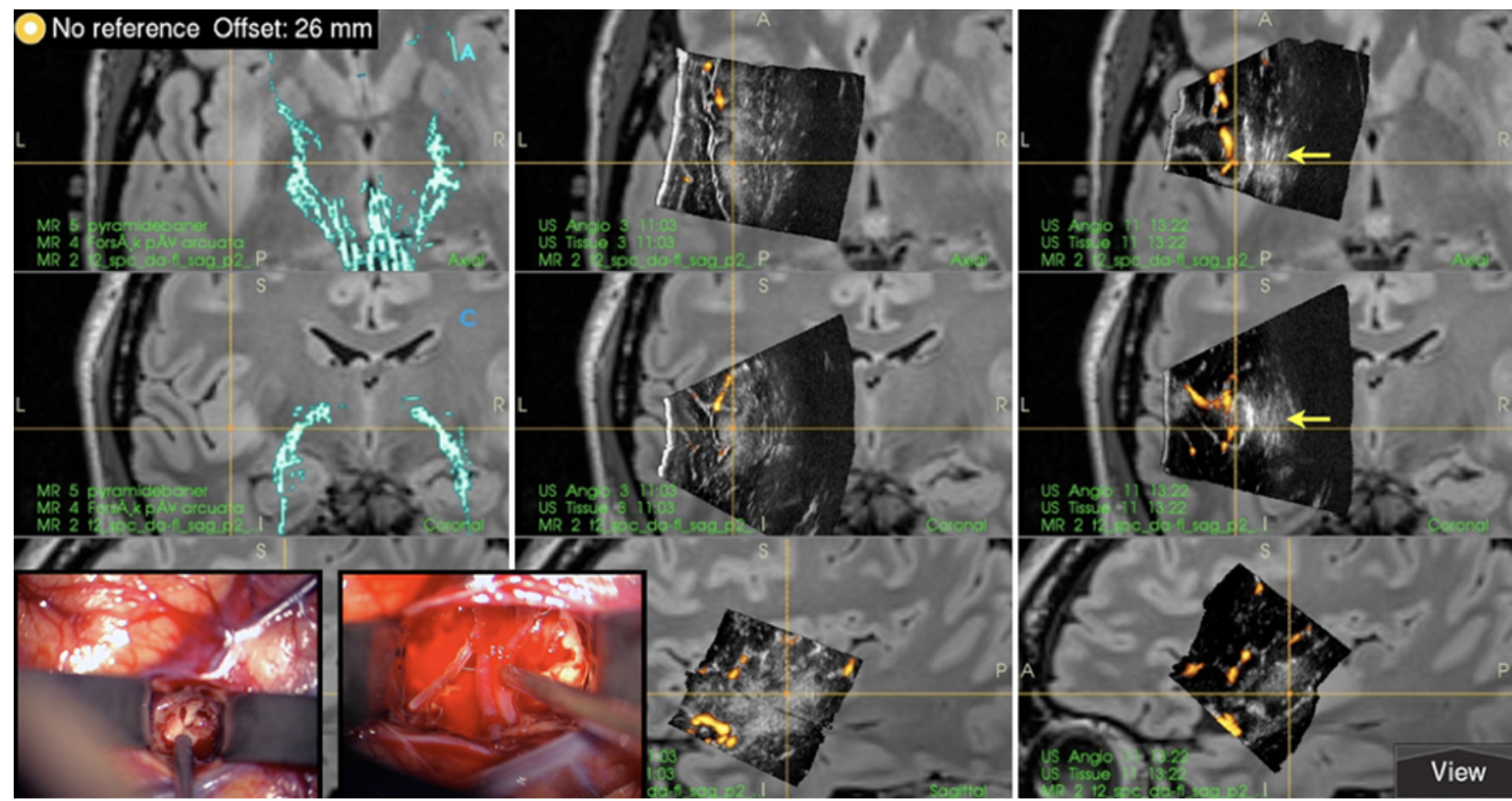

FIG. 4. Snapshots of the navigation monitor during surgery in patient 3 displayed in axial, coronal, and sagittal views (upper, center, and lower rows). The left column shows the MR images with pyramidal tract (turquoise), the center column shows the same cross section of the MR images with the corresponding cross sections from the 3D US acquired before the start of the tumor resection, and the right column shows the 3D US images at the end of the resection. No tumor tissue was visible at the end of the procedure. The power Doppler angiography (orange structures) depicts the insula arteries. The overlaid microscopic images show the surgical opening in the cortex (left) and the navigated pointer at the insula arteries (right). The cross sections of the MRI and 3D US volumes are steered by the position of this navigated pointer. The yellow arrows point at enhancement artifacts below the resection cavity.

relative to an updated map. Thus, it also enables the surgeon to keep a narrow access route through normal tissue. We have used tiny spatulas, but a tubular retractor would probably be useful as well.

One challenge with US imaging is the enhancement artifact that may appear toward the end of the operation. It was seen at the bottom of the operative cavity in patient 3. By comparing with previous US images, it is easy to see that these are artifacts. A new acoustic coupling fluid (ACF) has been developed that has the same damping effect as normal brain/tumor tissue and therefore will reduce/remove these artifacts. ${ }^{21}$ The fluid has not yet been tested in LGG operations, but it is easy to see the benefit in glioblastoma operations, as demonstrated in Fig. 5.

In patient 2, surgeons attempted to remove the tumor via the transsylvian approach at a local university hospital. This has been the traditional way of reaching the mesial part of the temporal lobe. ${ }^{23}$ With 3D US technology on hand, it is easier to use a transcortical approach. We go as basal as possible and always aim to keep the working channel through the normal tissue as narrow as possible. This is best achieved when the trajectory hits the center of the lesion. In that context, the precise localization of the lesion by 3D US is important, as it reduces the trauma to healthy brain and, in this way, possibly reduces morbidity. Tracts have to be avoided. With stimulation, as used in patients 1 and 2, the risk of entering the pyramidal tract is reduced, but stimulation would help neither with the anatomical orientation nor in identifying the tumor remnant; furthermore, stimulation would not allow for a precise approach in the way that guided US does. The benefits of intraoperative 3D US in overcoming these limitations of stimulation are probably most pronounced in deep-seated lesions when operating through a narrow corridor. A basal temporal access also reduces the risk for injury to the inferior frontooccipital fascicle, resulting in semantic paraphasia. ${ }^{5,11}$ In spite of the precise resection, patient 2 had a quadrantanopia found by perimetry after the operation, however, with no apparent impact on his daily life. Diffusion tensor imaging was not able to depict the optic radiation and Meyer's loop in our cases, which could have been useful to avoid visual field deficiency. ${ }^{20}$

Even though many still use the transsylvian approach for insular glioma operations, ${ }^{17}$ the direct approach may be beneficial. ${ }^{6}$ The added benefit of using 3D US for guidance is the possibility of making a very small opening in the cortex and the ability to precisely localize arteries by Doppler angiography, both the insular arteries (M2) and for the large tumors also the lenticulostriate arteries. ${ }^{19}$

\section{Conclusions}

3D US-guided LGG resection is a useful and cost-effective tool. When sufficient attention is given to the sur- 

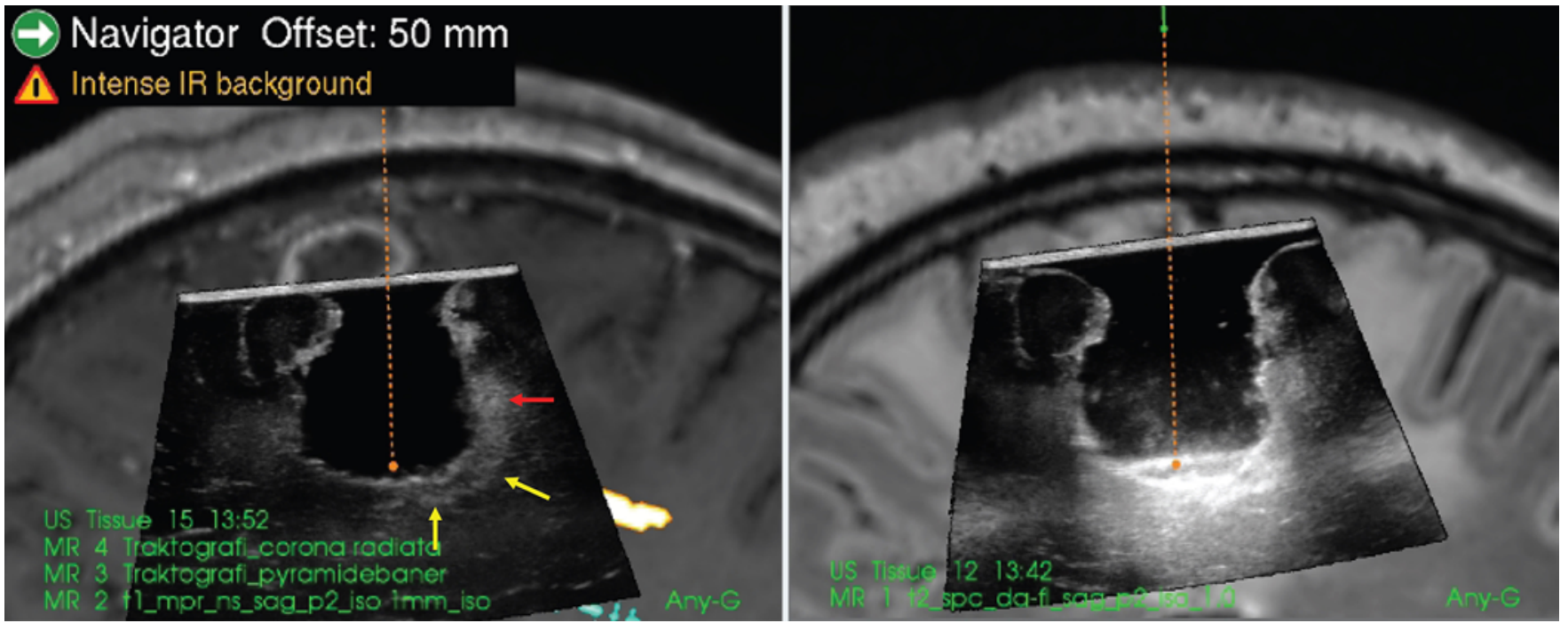

FIG. 5. Images from the study of an acoustic coupling fluid. Sections from 3D US images are overlaid on corresponding MR images. Left: Acoustic coupling fluid in the cavity during a glioblastoma operation. Right: Ringer's solution in the same operative cavity. Residual tumor indicated by yellow arrows in left image cannot be distinguished in right image, except for some suspected tumor tissue in the right wall (red arrow in left image) that can be seen in both images.

gical setup and patient positioning, 3D US-guided LGG surgery enables the surgeon to perform image-guided resections with high precision and with good image quality. It is a flexible tool with minimal interruption to normal workflow and can be used in every LGG procedure.

\section{Acknowledgments}

Tormod Selbekk is acknowledged for help with the preparation of the manuscript. Halvor Celius is acknowledged for language support.

\section{References}

1. Al-Otaibi F, Baeesa SS, Parrent AG, Girvin JP, Steven D: Surgical techniques for the treatment of temporal lobe epilepsy. Epilepsy Res Treat 2012:374848, 2012

2. Berntsen EM, Gulati S, Solheim O, Kvistad KA, Torp $\mathrm{SH}$, Selbekk T, et al: Functional magnetic resonance imaging and diffusion tensor tractography incorporated into an intraoperative 3-dimensional ultrasound-based neuronavigation system: impact on therapeutic strategies, extent of resection, and clinical outcome. Neurosurgery 67:251-264, 2010

3. Bø HKSO, Solheim O, Kvistad KA, Berntsen EM, Torp SH, Skjulsvik AJ, et al: Intraoperative 3D ultrasound-guided resection of diffuse low-grade gliomas: radiological and clinical results. J Neurosurg [epub ahead of print February 1, 2019. DOI: $10.3171 / 2018.10 . J N S 181290]$

4. Chang EF, Clark A, Smith JS, Polley MY, Chang SM, Barbaro NM, et al: Functional mapping-guided resection of low-grade gliomas in eloquent areas of the brain: improvement of long-term survival. Clinical article. J Neurosurg 114:566-573, 2011

5. De Benedictis A, Sarubbo S, Duffau H: Subcortical surgical anatomy of the lateral frontal region: human white matter dissection and correlations with functional insights provided by intraoperative direct brain stimulation: laboratory investigation. J Neurosurg 117:1053-1069, 2012
6. Hervey-Jumper SL, Li J, Osorio JA, Lau D, Molinaro AM, Benet A, et al: Surgical assessment of the insula. Part 2: validation of the Berger-Sanai zone classification system for predicting extent of glioma resection. J Neurosurg 124:482 488, 2016

7. Jakola AS, Myrmel KS, Kloster R, Torp SH, Lindal S, Unsgård $\mathrm{G}$, et al: Comparison of a strategy favoring early surgical resection vs a strategy favoring watchful waiting in lowgrade gliomas. JAMA 308:1881-1888, 2012

8. Jakola AS, Unsgård G, Myrmel KS, Kloster R, Torp SH, Losvik OK, et al: Surgical strategy in grade II astrocy toma: a population-based analysis of survival and morbidity with a strategy of early resection as compared to watchful waiting. Acta Neurochir (Wien) 155:2227-2235, 2013

9. Jakola AS, Unsgård G, Myrmel KS, Kloster R, Torp SH, Sagberg LM, et al: Surgical strategies in low-grade gliomas and implications for long-term quality of life. J Clin Neurosci 21:1304-1309, 2014

10. Kamada K, Todo T, Ota T, Ino K, Masutani Y, Aoki S, et al: The motor-evoked potential threshold evaluated by tractography and electrical stimulation. J Neurosurg 111:785-795, 2009

11. Kier EL, Staib LH, Davis LM, Bronen RA: MR imaging of the temporal stem: anatomic dissection tractography of the uncinate fasciculus, inferior occipitofrontal fasciculus, and Meyer's loop of the optic radiation. AJNR Am J Neuroradiol 25:677-691, 2004

12. Nossek E, Korn A, Shahar T, Kanner AA, Yaffe H, Marcovici $\mathrm{D}$, et al: Intraoperative mapping and monitoring of the corticospinal tracts with neurophysiological assessment and 3-dimensional ultrasonography-based navigation. Clinical article. J Neurosurg 114:738-746, 2011

13. Pallud J, Mandonnet E, Duffau H, Kujas M, Guillevin R, Galanaud D, et al: Prognostic value of initial magnetic resonance imaging growth rates for World Health Organization grade II gliomas. Ann Neurol 60:380-383, 2006

14. Ribas GC: The cerebral sulci and gyri. Neurosurg Focus 28(2):E2, 2010

15. Seidel K, Beck J, Stieglitz L, Schucht P, Raabe A: The warning-sign hierarchy between quantitative subcortical motor 
mapping and continuous motor evoked potential monitoring during resection of supratentorial brain tumors. J Neurosurg 118:287-296, 2013

16. Selbekk T, Jakola AS, Solheim O, Johansen TF, Lindseth F, Reinertsen I, et al: Ultrasound imaging in neurosurgery: approaches to minimize surgically induced image artefacts for improved resection control. Acta Neurochir (Wien) 155:973-980, 2013

17. Signorelli F, Guyotat J, Elisevich K, Barbagallo GM: Review of current microsurgical management of insular gliomas. Acta Neurochir (Wien) 152:19-26, 2010

18. Smith JS, Chang EF, Lamborn KR, Chang SM, Prados MD, Cha S, et al: Role of extent of resection in the long-term outcome of low-grade hemispheric gliomas. J Clin Oncol 26:1338-1345, 2008

19. Šteňo A, Jezberová M, Hollý V, Timárová G, Šteňo J: Visualization of lenticulostriate arteries during insular low-grade glioma surgeries by navigated 3D ultrasound power Doppler: technical note. J Neurosurg 125:1016-1023, 2016

20. Thudium MO, Campos AR, Urbach H, Clusmann H: The basal temporal approach for mesial temporal surgery: sparing the Meyer loop with navigated diffusion tensor tractography. Neurosurgery 67 (2 Suppl Operative):385-390, 2010

21. Unsgård G, Sagberg LM, Müller S, Selbekk T: A new acoustic coupling fluid with ability to reduce ultrasound imaging artefacts in brain tumour surgery-a phase I study. Acta Neurochir (Wien) 161:1475-1486, 2019

22. Wen HT, Rhoton AL Jr, de Oliveira E, Cardoso AC, Tedeschi $\mathrm{H}$, Baccanelli M, et al: Microsurgical anatomy of the temporal lobe: part 1: mesial temporal lobe anatomy and its vascular relationships as applied to amygdalohippocampectomy. Neurosurgery 45:549-592, 1999
23. Yaşargil MG, Teddy PJ, Roth P: Selective amygdalo-hippocampectomy. Operative anatomy and surgical technique. Adv Tech Stand Neurosurg 12:93-123, 1985

\section{Disclosures}

Dr. Unsgård reports holding a patent on the acoustic coupling fluid (ACF) EP2846841B1.

\section{Author Contributions}

Conception and design: Unsgård. Acquisition of data: both authors. Drafting the article: Unsgård. Critically revising the article: both authors. Reviewed submitted version of manuscript: Unsgård. Approved the final version of the manuscript on behalf of both authors: Unsgård. Administrative/technical/material support: Lindseth.

\section{Supplemental Information \\ Videos}

Video 1. https://vimeo.com/363551364.

Video 2. https://vimeo.com/363551392.

\section{Correspondence}

Geirmund Unsgård: St. Olav University Hospital, Norwegian University of Science and Technology, Trondheim, Norway. geirmund.unsgard@ntnu.no. 